\title{
Percolation Model for Proton Immobility in Ice
}

\author{
Mikrajuddin Abdullah* \\ Department of Physics, Institute of Technology, Bandung \\ Jalan Ganeca 10 Bandung 40132 Indonesia
}

\begin{abstract}
A simple Ising model and a statistical theory of gel formation in a polymer matrix were combined to explain the effect of temperature on the mobility of protons in ice. Cowin et al observed that protons in ice bulk were immobile at temperatures below $190 \mathrm{~K}$, and suddenly become mobile at temperatures above $190 \mathrm{~K}$ [Nature 398, 405 (1999)]. We proposed here that the transport of protons is controlled by a percolation mechanism. The proton transport is facilitated by the occurrence of easily reoriented water molecules in the ice bulk. The fraction of this molecule depends on temperature and was calculated using a simple Ising model. The formation of network of these molecules which provides pathways for proton transport was calculated using a statistical theory of gel formation in a polymeric system. Our model succeeded to reproduce the variation of potential difference between the ice film surfaces with respect to temperature as observed by Cowin et al based on a soft landing experiment.
\end{abstract}

Keywords: Ising model; gel theory of polymer; proton immobility; ice; percolation.

\section{$1 \quad$ Introduction}

Using a method of soft landing of hydronium ions on the surface of ice film, Cowin et al observed that protons were immobile in ice at temperatures below $190 \mathrm{~K}$ [1]. This observation seems to contradict the Grotthuss mechanism, whereby protons tunnel from one water molecule to the next $[2,3]$, which can persist even at $0 \mathrm{~K}[4,5]$. The reason proposed to explain this observation was the tunneling over long distances should only occur if the potential is periodic. Cowin et al argued that normal ice is proton disordered, thereby destroying the periodicity [1]. Another proposal was the self-trapping mechanism of hydronium ions in a polarization well. More specifically, a hydronium ion will force a nearby water molecule to reorientate, with their hydrogen ends pointing away from it. This results in a polarization pattern acting like a deep potential well that prevents the proton to escape.

\footnotetext{
${ }^{*}$ Corresponding author: Dr. Mikrajuddin Abdullah, Tel. +62-22-250-0834 Fax: +62-22-250-6452, E-mail din@fi.itb.ac.id.
}

Received May 27, 2005 . 
The data observed by Cowin et al is reproduced in Fig. 1 [1]. After "soft landing" deposition of hydronioum ions on the top surface of the ice film, electric potential difference between the top and the bottom surfaces of the film was measured. They concluded that the protons become immobile at temperatures below $190 \mathrm{~K}$. From the figure, we can see a sudden decrease in the potential difference at temperature around $150 \mathrm{~K}$ and $190 \mathrm{~K}$ for curves (a) and (b), respectively. It indicates that the resistivity of the film becomes very large at below $190 \mathrm{~K}$, and then decreases suddenly at $190 \mathrm{~K}$. This behavior is usually found in percolating systems. Therefore, it is challenging to examine the relation of this observation with the percolation mechanism. Until presently, no work has been reported to explain the proton transfer in ice using a percolation approach.

In this work, we describe the transport of proton in the ice film using a simple percolation model. As a starting point, we accepted the proposal of Cowin et al that proton transport is facilitated by the ability of the water molecule to reorientate [1]. We proposed that at low temperatures, only a small fraction of water molecules can reorientate easily. This fraction, however, increases with temperature. At certain temperature, this fraction is high enough to form a network of easily reoriented water molecules. This network serves as the pathways for proton transport, resulting in a sudden decrease in the potential difference between the film surfaces.

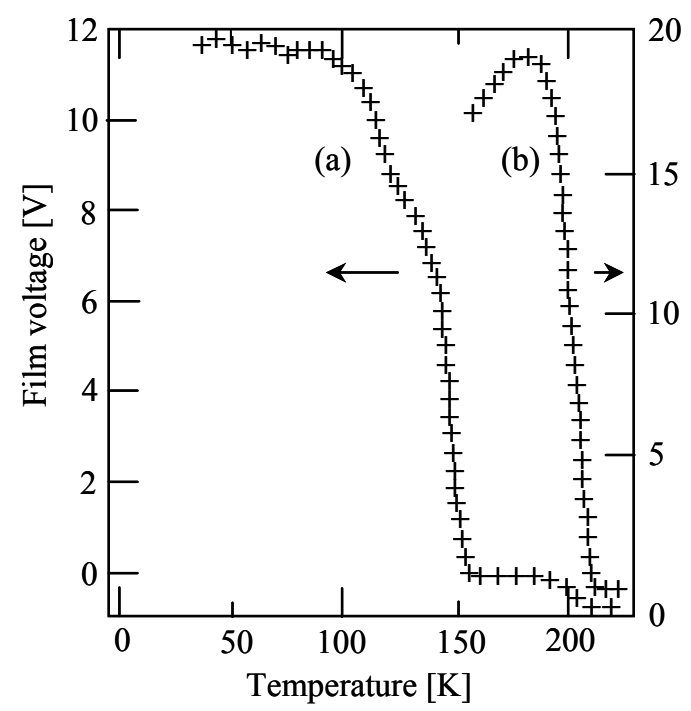

Figure 1 Effect of temperature on the potential difference between ice film surfaces. The data were adopted from Cowin et al [1]. Curve (a) was measured at low charge deposition and curve (b) was measured at high charge deposition. 
The problem to be solved is how to calculate the fraction of easily reoriented water molecules. To this aim, we used a simple Ising model. After finding this fraction, we calculated the fraction of these molecules that forms continuous networks, on the basis of a statistical theory of gel formation in polymeric systems. Combining the two approaches, we found a behavior very similar to that observed by Cowin et al [1].

\section{Theory}

\subsection{Model Development}

The basic assumptions of our model development are as follows:

1. The ice bulk is considered to consist of "easily reoriented (ER)" and "hardly reoriented (HR)" molecules.

2. Each molecule can be connected with $n$ nearest neighbor (each molecule has $n$ hands). In the connected ER molecule network, the proton can move from one molecule to another.

3. The range proton transport is related to the size of ER molecule network.

4. The fraction of ER molecules should depend on temperature, i.e., increases with temperature.

5. To predict the effect of temperature on the proton transport in the ice bulk, one needs to understand the development of the connected ER molecules. To this aim, we performed two step calculations of: (1) the fraction of ER molecules in the ice bulk using a simple Ising model, and (2) adopting the statistical theory of gel formation in a polymeric system to determine the fraction of connected ER molecules. We have also applied similar approach to predict the temperature dependence of electrical conductivity in polymer electrolytes [6] and porous silicon [7].

Suppose the total number of water molecules in the ice is $N$. We define the "upward" molecule and "downward" molecules according to their polarization states, somewhat similar to assignment of spin orientations that used in the Ising model, with a total number of $N_{+}$and $N_{-}$, respectively $\left(N=N_{+}+N_{-}\right)$. The ER water molecules correspond to molecules that can change easily their "orientation" from "upward" to "downward" and vice versa. On the other hand, the HR water molecules correspond to molecules that cannot easily change their "orientation" from "upward" to "downward" and vice versa. The average energy per molecule is approximated by

$$
\varepsilon\left(N_{+}, N\right)=-\frac{J}{N n} \sum_{i, j} \sigma_{i} \sigma_{j}
$$


having assumed the strength of interaction between "upward"-"upward", "downward"-"downward", and "upward"-"downward" molecules to be the same. In Eq. (1), $\sigma_{i}=+1$ is assigned to "upward" molecules and $\sigma_{i}=-1$ is assigned to "downward" molecules, $J$ is the strength of interaction between neighbor molecules, and $n$ is the number of nearest neighbors.

Using Bragg-William approximation, after defining the parameter of long-range order, $L$ which satisfies $N_{+} / N=(L+1) / 2$, we obtain the approximated form of the energy per molecule as $\varepsilon(L)=-(J / 2) L^{2}$, and the partition function is given approximately by [8]

$$
Q(T)=\sum_{L=-1}^{+1} \frac{N !}{\left[\frac{1}{2} N(1+L)\right] !\left[\frac{1}{2}(1-L)\right] !} \exp \left[\frac{N n J L^{2}}{2 k T}\right]
$$

As usual, assuming $N \rightarrow \infty$, we can approximate the factorial terms with a Stirling formula. Selecting only $\bar{L}$, i.e., the value of $L$ that maximizes the summation of Eq. (2), we obtain the final solution satisfied by $\bar{L}$ as

$$
\bar{L}=\tanh \left(\frac{n J \vec{L}}{k T}\right)
$$

The parameter $\bar{L}$ is called the ordering parameter, which can be associated with the fraction of the HR molecules. Therefore, the fraction of the ER molecules is expressed by

$$
\phi=1-\bar{L}
$$

Next, we consider the fraction of continuously connected ER molecules. We used the statistical theory of gel formation to determine this fraction. At low fraction of ER molecules, the molecules tend to disperse homogeneously in the ice bulk. No connected ER molecules is developed, so that the transport of proton is suppressed. At higher fraction of ER molecules, the ER molecule clusters are developed, the size of which increases with increasing fraction of ER molecules. At certain fraction of ER molecules (at a certain temperature), an infinitely connected ER molecules appears, resulting in a sudden increase in the proton conductivity. By further increasing the temperature (fraction of ER molecules), the infinitely connected ER molecules will dominate the ice bulk volume, along with decreasing the fraction of HR molecules. 


\subsection{Formation of Connected EF Molecules}

We define $\beta$ as the probability of direct bonding between two ER molecules. In a cluster of $m$ ER molecules (we call $m$-ER), some hands form bonds and the rest remain free. It requires the attachment of $(m-1)$ new ER molecules to a preselected ER molecule to form $m$-ER cluster [6]. If only one bond is created at each attachment of a new ER molecule, then $m$-1 bonds and $(n-2) m+1$ free hands (excluding the freely preselected hand) appears after the formation of $m$ ER cluster. Since the probability of a hand to form a bond is $\beta$ and to become free is $(1-\beta)$, the total probability for forming $m$-ER of any configuration is

$$
P_{m}=\Omega_{m} \beta^{m}(1-\beta)^{(n-2) m+1},
$$

with $\Omega_{m}$ is the total number of possible configurations.

To form $m-1$ bonds in an $m$-ER, we can select $m-1$ bonds from the total $(m-1) n$ bonds belonging to the added ER molecules in $[n(m-1)] ! /\{[(n-2) m+1] !(m-1) !\}$ distinct ways, and the added ER molecules can be attached sequentially in $(m-1)$ ! ways. Noting that since all ER molecules in $m$-ER cluster are identical, we have to add a dividing factor of $m$ ! and obtain

$$
\Omega_{m}=\frac{[n(m-1)] !}{[(n-2) m+1] !(m-1) !} \times \frac{(m-1) !}{m !}=\frac{[n(m-1)] !}{[(n-2) m+1] ! m !} .
$$

The number of free hands belonging to the $m$-ER (preselected ER molecule and the added ones) is $(n-2) m+2$. Suppose $N_{\mathrm{m}}$ is the population of $m$-ER and $N_{\mathrm{o}}$ is the total number of ER molecules. The number of free hands belonging to all $\mathrm{m}$ ER is $[(n-2) m+2] N_{m}$, the total number of hands belonging to all ER molecules is $N_{\mathrm{o}} n$, and the total number of free hands is $N_{\mathrm{o}} n(1-\beta)$. The alternative expression for $P_{m}$ is therefore given by [9],

$$
P_{m}=\frac{[(n-2) m+2] N_{m}}{N_{n} n(1-\beta)} .
$$

Combining (5) and (7) we obtain

$$
N_{m}=N_{o} n(1-\beta)^{2} \Omega_{m} \frac{\xi^{m}}{\beta}
$$


with $\xi=\beta(1-\beta)^{n-2}$. The volume of $m$-ER is

$$
W_{m}=m \frac{N_{m}}{N_{o}}=\frac{(1-\beta)^{2}}{\beta} n m \Omega_{m} \xi^{m},
$$

which satisfies the normalization condition

$$
\sum_{m=1}^{\infty} W_{m}=\sum_{m=1}^{\infty} \frac{(1-\beta)^{2}}{\beta} n m \Omega_{m} \xi^{m}=1
$$

The summation results in (10) depends on the $\xi$, and for a certain $\xi$, Eq. (10) gives only one summation result. Since $\xi$ is a polynomial of $\beta$, certain $\xi$ results in more than one $\beta$ s. For example, by replacing $\beta$ with $\beta^{*}$, which also satisfies $\xi=\beta^{*}\left(1-\beta^{*}\right)^{n-2}$, the summation results remains equal to unity. However, since in general $(1-\beta)^{2} / \beta \neq\left(1-\beta^{*}\right)^{2} / \beta^{*}$, changing $\beta$ with $\beta^{*}$ may results in different $\sum_{m} W_{m}$, which seems unphysical. The physical soundness is secured only when the smallest root of $\xi=\beta(1-\beta)^{n-2}$ is used [9]. Therefore, the accepted expression for $W_{\mathrm{m}}$ is

$$
W_{m}=\frac{\left(1-\beta^{*}\right)^{2}}{\beta^{*}} n m \Omega_{m} \xi^{m}
$$

with $\beta^{*}$ is the smallest root of $\beta(1-\beta)^{n-2}=\beta^{*}\left(1-\beta^{*}\right)^{n-2}$.

Summing $W_{\mathrm{m}}$ over all finite $m$ 's gives the fraction of the so called molecule sol (finite connected ER molecules) with respect to the fraction of ER molecules as

$$
W_{s}=\sum_{\text {all finitem }} W_{m}=\frac{(1-\beta)^{2} \beta^{*}}{\left(1-\beta^{*}\right)^{2} \beta} .
$$

The fraction of the gel (infinite connected ER molecules) with respect to the total fraction of ER molecules is therefore,

$$
W_{g}=1-W_{s}=1-\frac{(1-\beta)^{2} \beta^{*}}{\left(1-\beta^{*}\right)^{2} \beta} .
$$

The volume fraction of gel with respect to total volume of ice bulk becomes 


$$
W_{g}^{*}=\phi W_{g}
$$

\subsection{Effective Resistivity}

To determine the effective resistivity, we assume that the material is composed of two phases: (a) the ER gels and (b) a mixture of HR molecules and separated ER molecules. The resistivity of the first phase is denoted by $\rho_{1}$ and that of the second phase is denoted by $\rho_{2}$.

The resistivity of gels depends on the gel volume. If the average dimension of gel is $\ell$, the resistivity of gels can be approximated by

$$
\frac{1}{\rho_{g}} \propto \frac{1}{\rho_{1}}(A / \ell) \propto \frac{1}{\rho_{1}}\left(\ell^{2} / \ell\right) \propto \frac{1}{\rho_{1}} \sqrt[D]{W_{g}^{*}},
$$

with $D$ as the fractal dimension. We can write $\left(1 / \rho_{g}\right)=c\left(1 / \rho_{1}\right) \sqrt[D]{W_{g}^{*}}$, where $c$ is a constant. When $W_{g}^{*} \rightarrow 1, \rho_{g} \rightarrow \rho_{1}$, one finds $c=1$. The gels and the second phase form a parallel network, so the effective resistivity satisfies

$$
\frac{1}{\rho_{e}}=\frac{W_{g}^{*}}{\rho_{g}}+\frac{\left(1-W_{g}^{*}\right)}{\rho_{2}}=\frac{\left(W_{g}^{*}\right)^{1+1 / D}}{\rho_{1}}+\frac{\left(1-W_{g}^{*}\right)}{\rho_{2}}
$$

\section{$3 \quad$ Results and Discussion}

The probability of a hand to form a bond can be taken to be equal to the fraction of the ER molecules, or $\beta=\phi$ [6]. Normally, each hydrogen bond in the ice has four neighbors [1], therefore we used $n=4$ in the calculation. Figure 2 is the calculation result using $\rho_{2}=100 \rho_{1}$, with $\Delta V$ assumed to be proportional to $\rho_{e}$. The voltage difference, $\Delta V$, was then normalized to give a maximum value of 12 Volt. The gel was assumed to have a fractal dimension $D=2.5$. This value is close to the fractal dimensions of 2.57 to 2.87 for sandstone pores as observed by Katz and Thompson [10]. The behavior of the theoretical result fairly reproduces the behavior of the experimental data from Cowin et al [1]. The percolation-like transition of the potential difference was observed experimentally, which is also showed by the theoretical curve. 


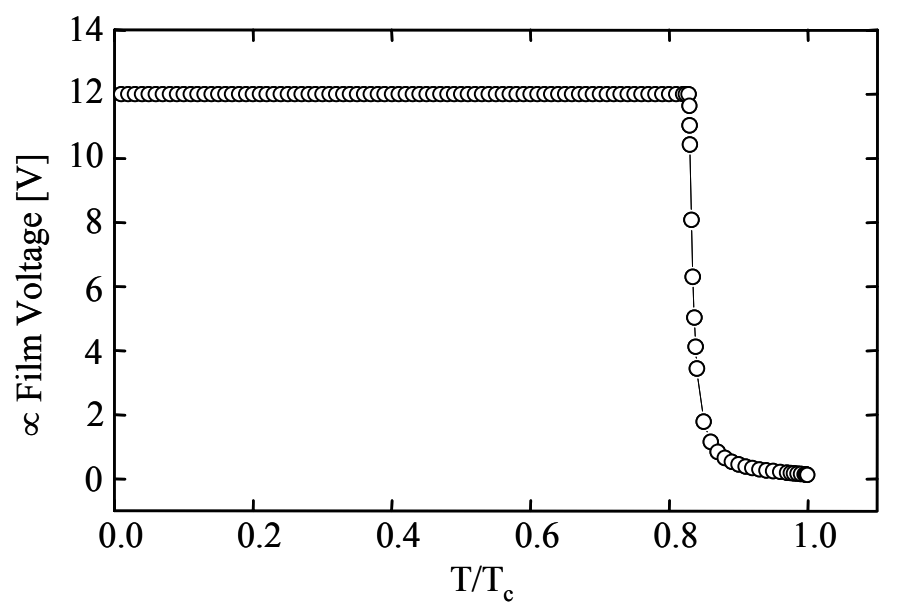

Figure 2 Theoretical curve showing the effect of temperature on the potential difference between ice film surfaces. For the calculation, the following parameters were used: $\rho_{2}=100 \rho_{1}, n=4, D=2.5$, and $\Delta V \propto \rho_{e}$.

From Fig. 2 we can see that the potential difference suddenly drops at $T / T_{c} \cong 0.828$. From the experimental data [1], the transition from high to low potential difference takes place at $190 \mathrm{~K}$. This result, therefore suggest a "Curie" temperature of $T_{c}=190 / 0.828=230 \mathrm{~K}$.

\section{Conclusion}

As a final remark we summarize that the simple Ising model and the statistical theory of gel formation in a polymeric systems can be used to explain the effect of temperature on the transport of protons in ice. The model succeeded in explaining the behavior of the measured data, which is likely to exhibit a percolation transition at certain temperature. The percolation transition was caused by the formation of the network of the easily reoriented (ER) water molecule to serve as the pathways for proton transport. The fraction of the ER water molecules was calculated by a simple Ising model, while the fraction of network of ER molecules was calculated using a statistical gel model. We also showed that the "Curie" temperature of the ice is around $230 \mathrm{~K}$.

\section{Acknowledgement}

Very nice correction of English from one reviewer (MOT) is gratefully acknowledged. 


\section{References}

1. Cowin, J. P., Tsekouras, A. A., Iedama, M. J., Wu, K. \& Ellison, G. B., Nature 398 (1999), p. 405.

2. Hobbs, P. V., Ice Physics (Clarendon, Oxford, 1974)

3. Whalley, E., Jpnes, S. J. \& Gold, L. W., Physics and Chemistry of Ice (Royal Soc. Canada, Ottawa, 1973)

4. Eckener, U., Helmreich, D. \& Engelhard, H., in Physics and Chemistry of Ice (eds E. Whalley, S. J. Jones, and L. W. Gold) 242-245 (Royal Soc. Canada, Ottawa, 1973).

5. $\quad$ Petrenko, V.F. \& Maeno, N., J. de Phys. C 48 (1987), p. 115.

6. Mikrajuddin, Indonesia J. Phys. 13 (2002), p. 222.

7. Mikrajuddin, Shi, F.G. \& Okuyama, K., Europhys. Lett. 54 (2001), p. 234.

8. Huang, K., Statistical Mechanics (John Wiley, New York, 1963).

9. Flory, P. J., Principles of Polymeric Chemistry (Cornel University Press, New York, 1956)

10. Katz, A. J. \& Thompson, A. H., Phys. Rev. Lett. 54 (1985), p. 1325. 